\title{
Two-Leg Ladders and Carbon Nanotubes: Exact Properties at Finite Doping
}

\author{
R. Konik ${ }^{1}$, F. Lesage ${ }^{2}$, A.W.W. Ludwig ${ }^{1}$, H. Saleur ${ }^{3}$. \\ ${ }^{1}$ Department of Physics, UCSB, Santa Barbara, CA 93106 \\ ${ }^{2}$ Centre de recherches mathématiques, Université de Montréal, C.P. 6128, Succ. centre-ville, Montréal H3C 3J7, Canada \\ ${ }^{3}$ Department of Physics, University of Southern California, Los-Angeles, CA 90089-0484
}

(October 1, 2018)

\begin{abstract}
Recently Lin, Balents, and Fisher have demonstrated that two-leg Hubbard ladders and armchair carbon nanotubes renormalize onto the integrable $\mathrm{SO}(8)$ Gross-Neveu model. We exploit this integrability to examine these systems in their doped phase. Using thermodynamic Bethe ansatz, we compute exactly both the spin and single particle gaps and the Luttinger parameter describing low energy excitations. We show both the spin gap and particle gap do not vanish at finite doping, while the Luttinger parameter remains close to its free fermionic value of 1 . A similar set of conclusions is drawn for the undoped systems' behaviour in a finite magnetic field. We also comment on the existence in these systems of the $\pi$-resonance, a hallmark of Zhang's SO(5) theory of high $T_{c}$ superconductivity.
\end{abstract}

Carbon nanotubes are among a new class of quasi one-dimensional materials exciting tremendous interest in both the material science and physics communities [1] [2] [3. Nanotubes are built by wrapping graphite sheets into tubes of nanometer scale. They take a multitude of forms, ranging from crystalline ropes of nanotubes arranged in triangular lattices to single multi-walled nanotubes consisting of concentric cylinders to the simplest construction, a single-walled, isolated nanotube. The latter promises to provide cleaner and longer realizations of quantum wires and as such promises to be the most relevant in new technologies [2].

Two-leg Hubbard ladders form another prominent class of quasi one-dimensional systems. Interest in these systems arises partly from the novel spin physics one expects in coupled chains [4, and partly in that they provide an example of a Mott-insulator, a class of compounds that includes undoped high $T_{c}$ cuprates. In contrast to the latter, here long-range antiferromagnetic order cannot occur and there is a finite gap to spin excitations. As such they represent a spin liquid. Their basic doped behaviour has been examined both through weak coupling analytical treatments [5] [7] and through Monte Carlo and density matrix renormalization group (DMRG) techniques [6]: the spin gap decreases in magnitude upon doping and the system exhibits quasi long range d-wave superconducting pairing correlations. We analyze this behaviour exactly in the following.

Remarkably a certain class of the single walled nanotubes behave as two-leg Hubbard ladders [3]. Single walled tubes are classified by a superlattice translation vector, denoted by $(\mathrm{N}, \mathrm{M})$, specifying how the graphite sheet is to be rolled up in forming the tube. "Armchair" tubes, described by a superlattice vector $(\mathrm{N}, \mathrm{N})$, have been shown to be identical to a two-leg ladder with an effective Hubbard interaction of $U / N$. Our findings for doped ladders thus apply equally to doped armchair nanotubes. Due to their relative cleanness, it may well be in such systems that our results are most readily observed. Electronic properties of the tubes can be easily measured by attaching metallic leads or by tunneling through an STM tip. The feasibility of the latter has been recently demonstrated by J. Wildöer et al. [1]. Exact results for the tunneling $I(V)$-curve into an infinitely long tube have recently been obtained by two of us [10].

Even more remarkable is the finding by Lin, Balents, and Fisher [7] that the two-leg Hubbard ladder, and thus the armchair nanotubes, renormalize in weak coupling onto an $\mathrm{SO}(8)$ Gross-Neveu model. This equivalence is established by considering generic interactions. Under a one-loop renormalization group flow, the interacting Hamiltonian flows onto an SO(8) Gross-Neveu model,

$$
\begin{aligned}
H=\int d x & \left(\sum _ { a = 1 } ^ { 4 } \left(\Psi_{a L}^{\dagger} i \partial_{x} \Psi_{a L}-\Psi_{a R}^{\dagger} i \partial_{x} \Psi_{a R}\right.\right. \\
& \left.\left.+g\left(\Psi_{a L}^{\dagger} \Psi_{a R}-\Psi_{a R}^{\dagger} \Psi_{a L}\right)^{2}\right)\right) .
\end{aligned}
$$

a theory of four interacting Dirac fermions, $\left(\psi_{R a}, \psi_{L a}\right)$.

$\mathrm{SO}(8)$ is an immense symmetry, usually found only in the domain of grand unified theories and supersymmetric strings [8]. In condensed matter systems we are usually limited to considering systems with charge conservation (U(1) symmetry) and perhaps rotational invariance (SU(2) symmetry). But we are doubly blessed. The $\mathrm{SO}(8)$ symmetry resides in an integrable model. Often integrable models are considered to be of little use in describing experiments in that they are highly tuned and thus are not expected to describe generic behavior in experimental systems. However here we avoid this difficulty: the system is attracted to the integrable $\mathrm{SO}(8)$ Gross-Neveu model under a renormalization group flow. A similar situation was found in the tunneling between $\nu=1 / 3$ quantum Hall edge states [9].

Integrability provides us with a tremendous amount of information. The key to organizing this information 
is the exact knowledge of the basis of eigenstates of the fully interacting system. There is a precise notion of a "particle" in an integrable system akin to fermi liquid theory. However unlike fermi liquid theory the particle is perfectly stable. There is no particle decay or production; all scattering processes are encoded in two particle scattering matrices, $S$.

Particles in the $\mathrm{SO}(8)$ Gross-Neveu model are organized into three octets and a set of 29 bosonic particles. One octet is of fundamental (Majorana) fermions and is directly related to the particle/hole excitations associated with the four Dirac fermions in eqn.(1D). The remaining two octets are of fermionic "kink" particles. The 29 bosons consist of a rank two anti-symmetric tensor with a single scalar boson, together forming a single representation of the $\mathrm{SO}(8)$ Yangian. The masses of these particle are generated dynamically through the Gross-Neveu interactions (the quartic term in eqn.(1i)). In terms of the ladder parameters, the mass scale, $m$, is governed by, $m \sim t e^{-t / U}$, where $t$ is the ladder bandwidth and $U$ is the typical coupling strength. The fermions all have mass, $m$, whereas the bosonic particles have mass, $\sqrt{3} m$.

Knowledge of these masses together with knowledge of their quantum numbers was used to make a variety of predictions in [7] with respect to the correlation functions at low temperature and zero doping. Largely qualitative, these results were extended in [10], where matrix elements were computed exactly in order to obtain exact forms for the correlators. Here we consider the doped system at zero temperature. Though more difficult theoretically, it is more interesting experimentally. Beyond a critical value of the chemical potential, the gap to chargetwo excitations goes to zero and the system becomes conducting.

To model the doping, we add a chemical potential term, $H_{Q}=-\mu Q$, to the Gross-Neveu Hamiltonian where $Q$ is the total electric charge. As $Q$ is conserved by the original Hamiltonian, this addition does not spoil integrability. Rather it only lifts the degeneracy of particles with differing charges. To determine the charge of the octet of fundamental (Majorana) fermions, we organize them into four Dirac fermions (only in doing this do they have well defined charges). Of the four, one carries electric charge 2, the so-called cooperon, and one carries spin $S_{z}=1$, the magnon. All four are associated with two-electron excitations. While the fundamental fermions carry but a single quantum number, the two octets of kink particles each carry $\pm 1 / 2$ of each of the four above quantum numbers. In particular, kinks carry charge, $Q= \pm 1$, and spin, $S_{z}= \pm 1 / 2$, and so a subset of them are identified with the physical electronic excitations on the ladder [7]. The 29 bosonic particles can be thought of as bound states of the fundamental fermions, and so carry two quantum numbers, for example, $Q=2$ and $S_{z}=1$.

As the chemical potential is turned on, a particle with charge $Q$ lowers its energy to $m-\mu Q$. As the energy of a particular particle becomes negative, the ground state of the system changes. Once empty, it now begins to fill with particles, much as if the fermi energy was moved above the bottom of the conduction band in a semiconductor. The first particle that will enter the ground state is the one with the highest charge to mass ratio. This is the cooperon. The ground state is pictured in Fig. 1 for $2 \mu>m$, where it consists solely of cooperons.
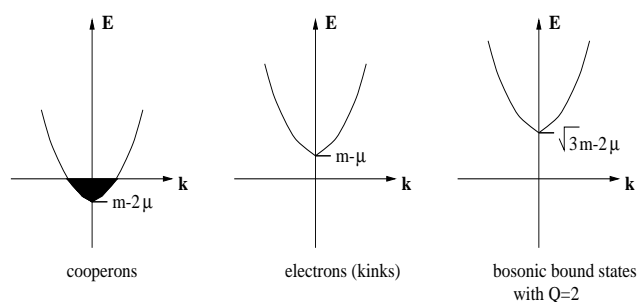

FIG. 1. Schematic of the doped ground state.

Seemingly as $\mu$ is increased further, the kinks and bosonic bound states will begin to fill into the ground state. However the increase in chemical potential is counteracted by the repulsion felt by the kinks and bound states to the cooperons. It turns out that this repulsive energy is greater than the chemical potential for arbitrary $\mu$, and the ground state remains solely filled with cooperons, as follows from the general results of [11]. We emphasize that this is an exact result.

We now proceed to characterize quantitatively the ground state through thermodynamic Bethe ansatz (TBA). At the root of the ansatz lies the insistence that the cooperon wave function be consistent with the scattering amplitudes, $S_{c c}$ (given in [12]), of two cooperons:

$S_{c c}(\theta)=\frac{\Gamma\left(1+\frac{i \theta}{2 \pi}\right) \Gamma\left(1 / 2-\frac{i \theta}{2 \pi}\right) \Gamma\left(5 / 6-\frac{i \theta}{2 \pi}\right) \Gamma\left(1 / 3+\frac{i \theta}{2 \pi}\right)}{\Gamma\left(1-\frac{i \theta}{2 \pi}\right) \Gamma\left(1 / 2+\frac{i \theta}{2 \pi}\right) \Gamma\left(5 / 6+\frac{i \theta}{2 \pi}\right) \Gamma\left(1 / 3-\frac{i \theta}{2 \pi}\right)}$.

In this way we can derive a density of occupied states for the cooperons, $\tilde{\rho}_{c}$, and a corresponding energy, $\epsilon_{c}$, of the cooperons. We parameterize the non-interacting energy and momentum of a particle of mass, $m$, by $E=m \cosh (\theta), p=m \sinh (\theta)$. With this parameterization, the use of periodic boundary conditions results in an equation which determines the density of occupied states per unit length for $|\theta|<B$ :

$$
\tilde{\rho}_{c}(\theta)=\frac{m}{2 \pi} \cosh (\theta)+\int_{-B}^{B} d \theta \tilde{\rho}_{c}\left(\theta^{\prime}\right) \Gamma_{c c}\left(\theta-\theta^{\prime}\right) .
$$

Here $\Gamma_{c c}=\frac{1}{2 \pi i} \partial_{\theta} \ln S_{c c}$ is a measure of the strength of the cooperon-cooperon interaction; if $\Gamma_{c c}=0$ we recover the density of occupied states for free fermions. $\pm B$ marks out the fermi energy. For $|\theta|>B, \tilde{\rho}_{c}(\theta)=0$. One determines the interacting cooperon energy, $\epsilon_{c}(\theta)$, by associating it with the occupied density of states at $T \neq 0$ via $\tilde{\rho}_{c} \equiv 1 /\left(1+\exp \left(\epsilon_{c} / T\right)\right)$. Demanding that the free en- 
ergy be minimized and then taking $T \rightarrow 0$, one obtains $\epsilon_{c}$ in terms of the cooperon-cooperon S-matrix:

$$
\epsilon_{c}(\theta)-\int_{-B}^{B} d \theta^{\prime} \Gamma_{c c}\left(\theta-\theta^{\prime}\right) \epsilon_{c}\left(\theta^{\prime}\right)=m \cosh (\theta)-2 \mu .
$$

Solving the constraint, $\epsilon_{c}(B)=0$, determines $B$.

Having described the ground state, we now look at excitations over the ground state, that is the gaps. We have already seen that the gap to a charge-two excitation is zero; we can add a cooperon at an infinitesmial energy above the fermi energy. More interesting are the gaps to spin 1 and single particle excitations. As emphasized above, these gaps never vanish at finite doping, a rigourous result.

We first consider the spin gap, $\Delta_{s}$. To compute this gap we want to identify the $\mathrm{Q}=0, S_{z}=1$ excitations. There are two possibilities: 1) adding a $Q=0, S_{z}=1$ magnon; and 2) removing a cooperon from the sea and adding a bosonic bound-state with $Q=2$ and $S_{z}=1$. For $2 \mu<m$, the first excitation is the only one possible; the sea is empty. The spin gap is then $\Delta_{s}=m$. As we cross the threshold, $2 \mu=m$, the Fermi sea begins to fill. At the threshold, the second process has a gap of only $m(\sqrt{3}-1)$, the energy of the cooperon hole added to the energy of the bound state [7]. It is thus lower than the first process, and the spin gap is determined by this excitation. We can push beyond threshold and compute the gap for all values of the doping: previous studies 12 13. have shown how to obtain the exact ground state of the theory at finite doping. The lowest $S_{z}=1, Q=0$ excitation is given by removing a cooperon at the fermi surface with rapidity, $\theta_{h}=B$, and adding a bound state with rapidity $\theta_{b}=0$. With these changes the particle density is modified to $\tilde{\rho}_{c}\left(\theta \mid \theta^{h}, \theta^{b}\right)$. $\tilde{\rho}_{c}\left(\theta \mid \theta^{h}, \theta^{b}\right)$ is determined by an equation similar to (3),

$$
\begin{array}{r}
\tilde{\rho}_{c}\left(\theta \mid \theta_{h}, \theta_{b}\right)-\int_{-B}^{B} d \theta^{\prime} \Gamma_{c c}\left(\theta-\theta^{\prime}\right) \tilde{\rho}_{c}\left(\theta^{\prime} \mid \theta_{h}, \theta_{b}\right)= \\
\Gamma_{c b}\left(\theta-\theta_{b}\right)-\Gamma_{c c}\left(\theta-\theta_{h}\right) .
\end{array}
$$

Here we have used $\Gamma_{c b}=\frac{1}{2 \pi i} \partial_{\theta} \ln S_{c b}$, where

$$
S_{c b}(\theta)=S_{c c}\left(\theta-\frac{i \pi}{6}\right) S_{c c}\left(\theta+\frac{i \pi}{6}\right) \frac{\theta+\frac{i \pi}{6}}{\theta-\frac{i \pi}{6}} .
$$

$S_{c b}$ is the cooperon-bound state S-matrix [10] [14].

Having the new density, the energy of the excited state is then straightforward to compute: it is determined by the difference between the energy of the excited system and the energy of the original ground state. Given that the energy of the cooperon sea is $L \int d \theta \tilde{\rho}_{c}(\theta)(\cosh (\theta)-$ $2 \mu / m)$, the spin gap equals

$$
\frac{\Delta_{s}}{m}=\sqrt{3}-\cosh B+\int_{-B}^{B} d \theta \delta \tilde{\rho}_{c}(\theta)\left(\cosh \theta-2 \frac{\mu}{m}\right)
$$

where $\delta \tilde{\rho}_{c}(\theta)=\tilde{\rho}_{c}\left(\theta \mid \theta_{h}, \theta_{b}\right)-\tilde{\rho}_{c}(\theta)$.

The single particle gap, defined as the energy to add an electron with $\mathrm{Q}=1$ (i.e. a kink) to the system, follows from similar considerations. For $2 \mu<m$, the gap is that of adding a kink in the absence of a fermi sea, $\Delta_{f}=m-\mu$. Crossing the threshold, $2 \mu>m$, the sea begins to fill in and the interactions between the kink and the cooperons must be taken into account. The scattering between the cooperons and kinks, $S_{c k}$ is given by

$$
S_{c k}(\theta)=\frac{\theta-\frac{i 2 \pi}{3}}{\theta+\frac{i 2 \pi}{3}} \frac{s(\theta)+\frac{i \sqrt{3}}{2}}{s(\theta)-\frac{i \sqrt{3}}{2}} \frac{\Gamma\left(\frac{5}{6}+\frac{i \theta}{2 \pi}\right) \Gamma\left(\frac{4}{3}-\frac{i \theta}{2 \pi}\right)}{\Gamma\left(\frac{5}{6}-\frac{i \theta}{2 \pi}\right) \Gamma\left(\frac{4}{3}+\frac{i \theta}{2 \pi}\right)},
$$

with $s(\theta)=\sinh (\theta)$. The same procedure as before leads to the single particle gap, $\Delta_{f}$ :

$$
\frac{\Delta_{f}}{m}=1-\frac{\mu}{m}+\int_{-B}^{B} d \theta \delta \tilde{\rho}_{c}(\theta)\left(\cosh (\theta)-2 \frac{\mu}{m}\right) .
$$

Here $\delta \tilde{\rho}_{c}$ is given by removing $\Gamma_{c c}$ and replacing $\Gamma_{c b}$ by $\Gamma_{c k}=\partial_{\theta} \ln S_{c k} / 2 \pi i$, both on the r.h.s. of eqn. (5).

Both of these gaps are shown in Fig. 2. The gaps have been plotted vs. $D=\int d \theta \tilde{\rho}_{c}(\theta)$, the density of cooperons (that is, the carrier density). We observe that there is a uniform decrease with increasing doping, as expected for this system. For large $D / m$, the gaps approach zero asymptotically without crossing, as can be established analytically.

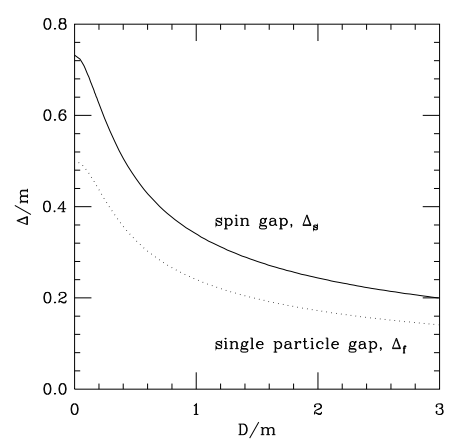

FIG. 2. Plot of the spin and single particle gap.

Although the gap to charge two excitations is zero, these excitations still encode interesting physics. The massless charge excitations occur near the fermi rapidity, $\theta= \pm B$. These excitations can be described by two chiral bosons, $\phi_{L / R}$, (one for each fermi point). That the cooperon sea is interacting is reflected in the Luttinger parameter, $K$, that governs these massless bosonic excitations. The propagator of $\phi_{L / R}$ is given in terms of $K$. Focusing on $\phi_{L}$ we have, $\left\langle\phi_{L}(z) \phi_{L}(w)\right\rangle=-K \ln (z-w)$. The operator creating a Cooper pair is thus $O_{c}=e^{i \phi_{L} / K}$, and so is governed by the propagator

$$
\left\langle O_{c}(z) O_{c}(w)\right\rangle=(z-w)^{-\frac{1}{K}}
$$

where $z=v_{F} \tau+i x$. $K$ thus describes the long distance asymptotics of this correlator. 
We can easily determine $\mathrm{K}$ in terms of the renormalized charge, $Q_{R}=-\left.\partial_{\mu} \epsilon_{c}\right|_{\theta=B} / 2 \equiv Z$, of the elementary excitations near the fermi surface. The basic idea, discussed at length in [15], is that the low energy excitations near the Fermi rapidity are just free fermions - that is, the dressed $\mathrm{S}$ matrix at low energy is just -1 . This means that the only non vanishing matrix elements of the current operator, $j_{L}=\partial_{z} \phi_{L}$, in this basis involve particle-hole pairs, which, parameterizing the energy of the massless excitations by $E(\theta)=e^{\theta}$, are given by,

$$
\left\langle\theta_{\text {hole }}, \theta_{\text {particle }}\left|j_{L}(0)\right| 0\right\rangle=c e^{\theta_{\text {hole }} / 2} e^{\theta_{\text {particle }} / 2} .
$$

The constant, $c$, is related to the interaction parameter, $K$, as we expect $\left\langle j_{L}(z) j_{L}(0)\right\rangle=-K / z^{2}$. Using the matrix element to compute this correlator, we find $K=-c^{2} / 4 \pi^{2}$. On the other hand, $c$ is related via $c=i 2 \pi Q_{R}$ to the renormalized charge, since integrating (11) over all $x$ gives the value of $Q_{R}$ on the one particle states. One thus finds $K=Q_{R}^{2}=Z^{2}$. $K$, using eqn. $(4)$, is then readily computed with the results plotted in Figure 3 . With $K<1$, the effective interactions are repulsive but because the cooperon-cooperon interaction is weak, the Luttinger parameter does not vary significantly from its free fermionic value of $K=1$. Interestingly, the plot is marked by a dip at about $D / m \sim .4$ before beginning an approach (albeit slowly) to an asymptotic value of $K=1$.

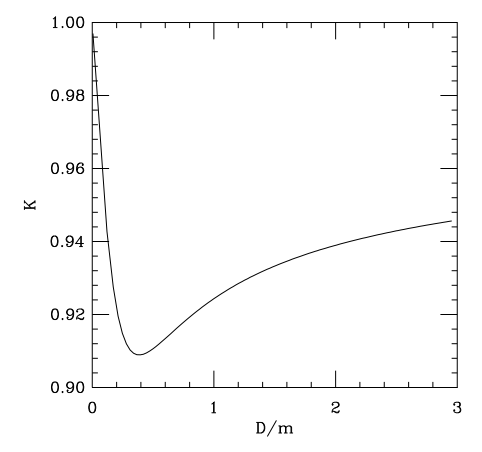

FIG. 3. Plot of the Luttinger parameter.

We point out that all of the above analysis applies equally to the system in a magnetic field, $H$, at zero doping. Rather than coupling to the cooperon, the magnetic field couples to the magnon. However as the magnon and cooperon are related by an $\mathrm{SO}(8)$ rotation, all of the above equations hold under relabeling. In this scenario, the ground state consists of interacting magnons with zero spin gap. $\Delta_{s}$ becomes the charge gap while $\Delta_{f}$ remains the gap to single particle excitations (with $S_{z}=1$ ). Here $K$ describes the low energy spin 1 excitations about the fermi surface.

To conclude, we would like to make a few comments about the equivalent of the " $\pi$ resonance" discussed in Zhang [16]. Understanding singularities quantitatively is beyond the scope of this note; however, certain simple threshold effects can easily be analyzed. In particular, let us consider the spin spectral function. This can be computed, at least in principle, by inserting a complete set of states between the two spin operators, corresponding to various physical processes. We consider in particular spin excitations with energy $2 \mu$. Such excitations can be obtained by applying Zhang's $\pi$-operator to low energy $Q=2$ excitations. The $Q=2$ excitations are formed by adding a single cooperon to the ground state directly above the fermi sea together with cooperon particle-hole pairs. As there are an infinite number of such excitations, we expect the spin-spin correlator to exhibit, at momentum $(\pi, \pi)$, a singularity of the type $|\omega-2 \mu|^{x} \Theta(\omega-2 \mu)$, akin to that found in [7]. However unlike [7] we expect this singularity to be robust: with integrability, massive excitations well above the continuum can still be stable because they are protected by higher order conserved quantities preventing their decay, although clearly, non-integrable perturbations will give these excitations a small finite lifetime.

The authors would like to acknowledge discussions with H.H.Lin, L. Balents, M.P.A. Fisher, and D. Duffy. This work has been supported by NSERC and the National Science Foundation through the Waterman Award under grant number DMR-9528578 (R.K.), by the A.P. Sloan Foundation (A.W.W.L.), the Packard Foundation, the NYI Program, and the DOE (H.S.).

[1] T. Ebbeson, Physics Today 49 (1996) 26; C. Kane and E.J. Mele, cond-mat/9608146; R. Egger and A. Gogolin, cond-mat/9708065; ibid., cond-mat/9803128; M. S. Dresselhaus, Nature 391 (1998) 19; M. Bockrath, D. Cobden, P. McEuen, N. Chopra, A. Zettl, A. Thess, and R. Smalley, Science 275 (1997) 1922; A. Rao, E. Richter, S. Bandow, B. Chase, P. Eklund, K. Williams, S. Fang, K. Subbaswamy, M. Menon, A. Thess, R. Smalley, G. Dresselhaus, and M. Dresselhaus, Science 275 (1997) 187; J. Wildöer, L. Venema, A. Rinzler, R. Smalley, and C. Dekker, Nature 391 (1998) 59.

[2] C. Kane, L. Balents, and M.P.A. Fisher, condmat/9708054.

[3] L. Balents and M.P.A. Fisher, cond-mat/9611126.

[4] E. Dagotto and T. Rice, Science 271 (1996) 618.

[5] M. Fabrizio, Phys. Rev. B 48 (1993) 15838; H. Kuroki and H. Aoki, Phys. Rev. Lett 72 (1994) 2947; H. Schulz, Phys. Rev. B 53 R (1996) 2959; L. Balents and M.P.A. Fisher, Phys. Rev. B 53 (1996) 12133; H.H. Lin, L. Balents, and M. Fisher, Phys. Rev. B 56 (1997) 6569.

[6] R. Noack, D. J. Scalapino, and S. White, Phil. Mag. B 74 (1996) 485; R. Noack, S. White, and D. Scalapino, Physica C 270 (1996) 281; R. Noack, S. White, and D. Scalapino, Europhys. Lett. 30 (1995) 163.; D. Duffy, S. 
Haas, and E. Kim cond-mat/9804221.

[7] H.H. Lin, L. Balents, M.P.A. Fisher, cond-mat/9801285.

[8] M. Green, J. Schwarz, and E. Witten, Superstring Theory, Cambridge University Press, Cambridge, 1987.

[9] P. Fendley, A.W.W. Ludwig, and H. Saleur, Phys. Rev. Lett 74 (1995) 3005; ibid. 75 (1995) 2196; Phys. Rev. B 52 (1995) 8934.

[10] R. M. Konik and A.W.W. Ludwig, to appear shortly.

[11] J. Evans and T. Hollowood, Nucl. Phys. Proc. Suppl. 45A (1996) 130; T. Hollowood and J. Evans, unpublished.

[12] P. Forgács, F. Niedermaier, P. Weisz,Nucl. Phys. B367, (1991) 123.

[13] G. Japaridze, A. Nersesyan, and P. Wiegmann, Nucl. Phys. B 230 (1984) 511.

[14] M. Karowski, H.J. Thun, Nucl. Phys. B190,(1981) 61.

[15] F. Lesage, H. Saleur, Nucl. Phys. B490 [FS] (1997) 543.

[16] E. Demler and S.C. Zhang, cond-mat/9502060. 